\title{
The DASH Prototype: Implementation and Performance
}

\author{
Daniel Lenoski, James Laudon, Truman Joe, David Nakahira, \\ Luis Stevens, Anoop Gupta and John Hennessy
}

Computer Systems Laboratory
Stanford University, CA 94305

\begin{abstract}
The fundamental premise behind the DASH project is that it is feasible to build large-scale shared-memory multiprocessors with hardware cache coherence. While paper studies and software simulators are useful for understanding many high-level design tradeoffs, prototypes are essential to ensure that no critical details are overlooked. A prototype provides convincing evidence of the feasibility of the design, allows one to accurately estimate both the hardware and the complexity cost of various features, and provides a platform for studying real workloads. A 16-processor prototype of the DASH multiprocessor has been operational for the last six months. In this paper, the hardware overhead of directory-based cache coherence in the prototype is examined. We also discuss the performance of the system, and the speedups obtained by parallel applications running on the prototype. Using a sophisticated hardware performance monitor, we characterize the effectiveness of coherent caches and the relationship between an application's reference behavior and its speedup.
\end{abstract}

\subsection{Introduction}

For parallel architectures to achieve widespread usage it is important that they efficiently nun a wide variery of applications without excessive programming difficulty. To maximize both high performance and wide applicability, we believe a parallel architecure should provide (i) the ability to support hundreds to thousands of processors, (ii) high-performance individual processors, and (iii) a single shared address space.

One important question that arises in the design of such large-scale singie-address-space machines is whether or not to allow caching of shared writeable data. The advantage, of course, is that caching allows higher performance to be achieved by reducing memory latency; the disadvantage is the problem of cache coherence. While solutions to the cache coherence problem are well understood for small-scale multiprocessors, they are unfortunately not so clear for large-scale machines. In fact, large-scale machines currentiy do not support cache coherence, and it has not been clear what the benefits and costs will be.

For the past several years, the DASH (Directory Architecture for SHared memory) project has been exploring the feasibility of

Permission to copy without fee all or part of this material is granted provided that the copies are not made or distributed for direct commercial advantage. the ACM copyright notice and the title of the publication and its date appear, and notice is given that copying is by permission of the Associanion for Computing Machinery. To copy otherwise, or to republish. requires a fee andior specific permission. building large-scale single-address-space machines with coherent caches. The key ideas are to distribute the main memory among the processing nodes to provide scalable memory bandwidth, and to use a distributed directory-based protocol to support cache coherence. To evaluate these ideas, we have constructed a prototype DASH machine. The full prototype will consist of sixty-four $33 \mathrm{MHz}$ MIPS R3000/R3010 processors, delivering up to 1600 MIPS and 600 scalar MFLOPS. An initial 16-processor prototype has been working for the past several months. and we are currently expanding this to the full 64-processor configuration.

This paper examines the hardware cost and performance characteristics of the prototype DASH system. Cost is measured in terms of the logic gates and the bytes of dynamic and static memory in the base system and the added directory logic. Performance is measured in terms of memory system bandwidth and latency, and in terms of parallel application speedups. For a representative set of the measured applications, we also present detailed reference statistics and relate these statistics to the observed application speedups. Finally, we describe the structure of the performance monitor logic which was used to take the detailed reference measurements.

The paper is organized as follows. Section 2 gives an overview of the DASH architecture. Section 3 introduces the DASH prototype and describes the logic used for the directory-based coherence protocol. Section 4 details the hardware costs of the system. Section 5 outlines the structure and function of the performance monitor logic, and Section 6 presents the performance of the memory system. and the speedups obtained by paraliel applications running on the prototype. We conclude in Section 7 with a summary of our experience with the DASH prototype.

\subsection{The DASH Architecture}

The DASH architecture has a two-level structure shown in Figure 1. At the top level, the architecture consists of a set of processing nodes (clusters) connected through a mesh interconnection network. In turn, each processing node is a bus-based multiprocessor. Intra-cluster cache coherence is implemented using a snoopy bus-based protocol, while inter-cluster coherence is maintained through a distributed directory-based protocol.

The cluster functions as a high-performance processing node. In addition. the grouping of multiple processors on a bus within each cluster amortizes the cost of the directory logic and the network interface. This grouping also reduces the directory memory requirements by keeping track of cached lines at a cluster as opposed to processor level. (We will more concretely discuss the role of clustering in reducing overhead in Section 4). 


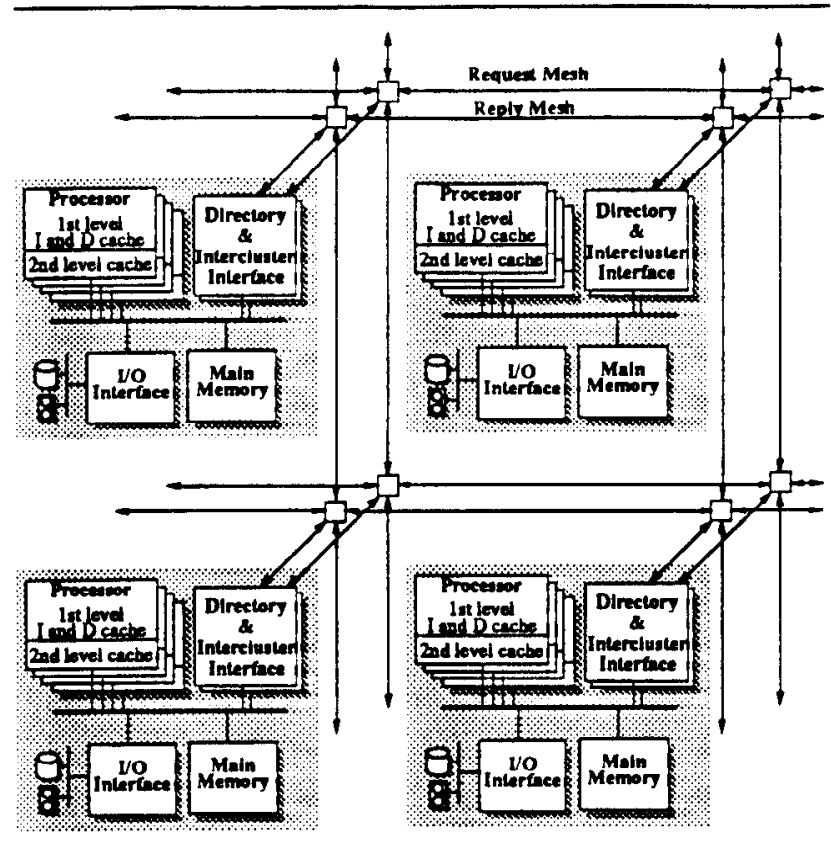

Figure 1. Block diagram of a $2 \times 2$ DASH prototype.

The directory-based protocol implements an invalidation-based coherence scheme. A memory location may be in one of three states: uncached, that is not cached by any processing node at all; shared. that is in an unmodified state in the caches of one or more nodes; or dirty, that is modified in the cache of some individual node. The directory keeps the summary information for each memory line, specifying the clusters that are caching it.

The DASH memory system can be logically broken into the four level hierarchy shown in Figure 2. The level closest to the processor is the processor cache and is designed to match the speed of the processor. A request that cannot be serviced by the processor cache is sent to the second level in the hierarchy, the local cluster level. This level consists of other processors' caches within the requesting processor's cluster. If the data is locally cached, the request can be serviced within the cluster, otherwise the request is sent to the directory home level. The home level consists of the cluster that contains the directory and physical memory for a given memory address. For some addresses, the local and home cluster are the same and the second and third level access occur simultaneously. In general, however, the request will travel through the interconnect to the home cluster. The home cluster can usually satisfy a request, but if the directory entry is in the dirty state, or in the shared state when the requesting processor requires exclusive access, the fourth, remote cluster level, must be accessed. The remole cluster level responds directly to the local cluster level while also updating the directory level.

In addition to providing coherent caches to reduce memory latency, DASH supports several other techniques for hiding and tolerating memory latency. DASH supports the release consistency model, that helps hide latency by allowing buffering and pipelining among memory requests. DASH also supports sofiware-controlled non-binding prefetching to help hide latency of read operations. Finally. DASH supports efficient spin locks in hardware and fetch-and-incr/decr primitives to help reduce the overhead of synchronization. Since we will primarily be focussing on the basic cache coherence protocol in this paper, we will not

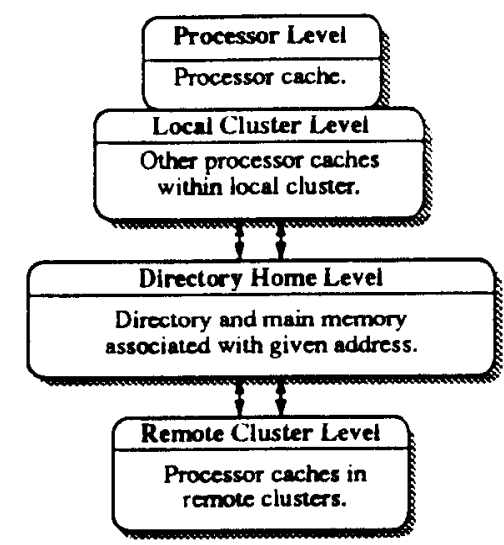

Figure 2. Logical memory hierarchy of DASH.

describe the details of these optimizations. For a more detailed discussion of the protocol and the optimizations, see $[7,8]$.

\subsection{The DASH Prototype}

To focus our effor on the novel aspects of the design and speed completion of a usable system, the base cluster hardware of the prototype is a commercially availabie bus-based multiprocessor. While there are some constraints and compromises imposed by the given hardware, the prototype still makes an interesting research vehicle.

The prototype system is based on a Silicon Graphics POWER Station $4 D / 340$ as the base cluster [3]. The $4 D / 340$ system consists of four MIPS R3000 processors and R3010 floating-point coprocessors running at $33 \mathrm{MHz}$. Each R3000/R3010 combination can achieve execution rates up to 25 VAX MIPS and 10 MFLOPS. Each CPU contains a 64 Kbyte instruction cache and a 64 Kbyte write-through data cache. The $64 \mathrm{Kbyte}$ data cache interfaces to a 256 Kbyte second-level write-back cache. The interface consists of a read buffer and a 4 word deep write-buffer. Both the first and second-level caches are direct-mapped and support 16 byte lines. The first-level caches run synchronously to their associated $33 \mathrm{MHz}$ processors while the second-level caches run synchronous to an independent $16 \mathrm{MHz}$ memory bus clock.

The second-level processor caches are responsible for bus snooping and maintaining coherence among the caches in the cluster. Since the first-level caches satisfy most memory requests, the second-level caches do not need duplicate snooping tags. Coherence is maintained with a MESI (Illinois) protocol[12], and inclusion of the first-level cache by the second-level. The main advantage of using the Illinois protocol in DASH is the cache-to-cache transfers specified in this protocol. While they do little to reduce the latency for misses serviced by local memory, local cache-to-cache transfers can greatly reduce the penalty for remote memory misses. The set of processor caches effectively act as a cluster cache for remote memory.

The memory bus (MPBUS) of the $4 D / 340$ is a synchronous bus and consists of separate 32-bit address and 64-bit data buses. The MPBUS is pipelined and supports memory-to-cache and cache-tocache transfers of 16 bytes every 4 bus clocks with a latency of 6 bus clocks. This results in a maximum bandwidth of $64 \mathrm{Mbytes} / \mathrm{sec}$. 


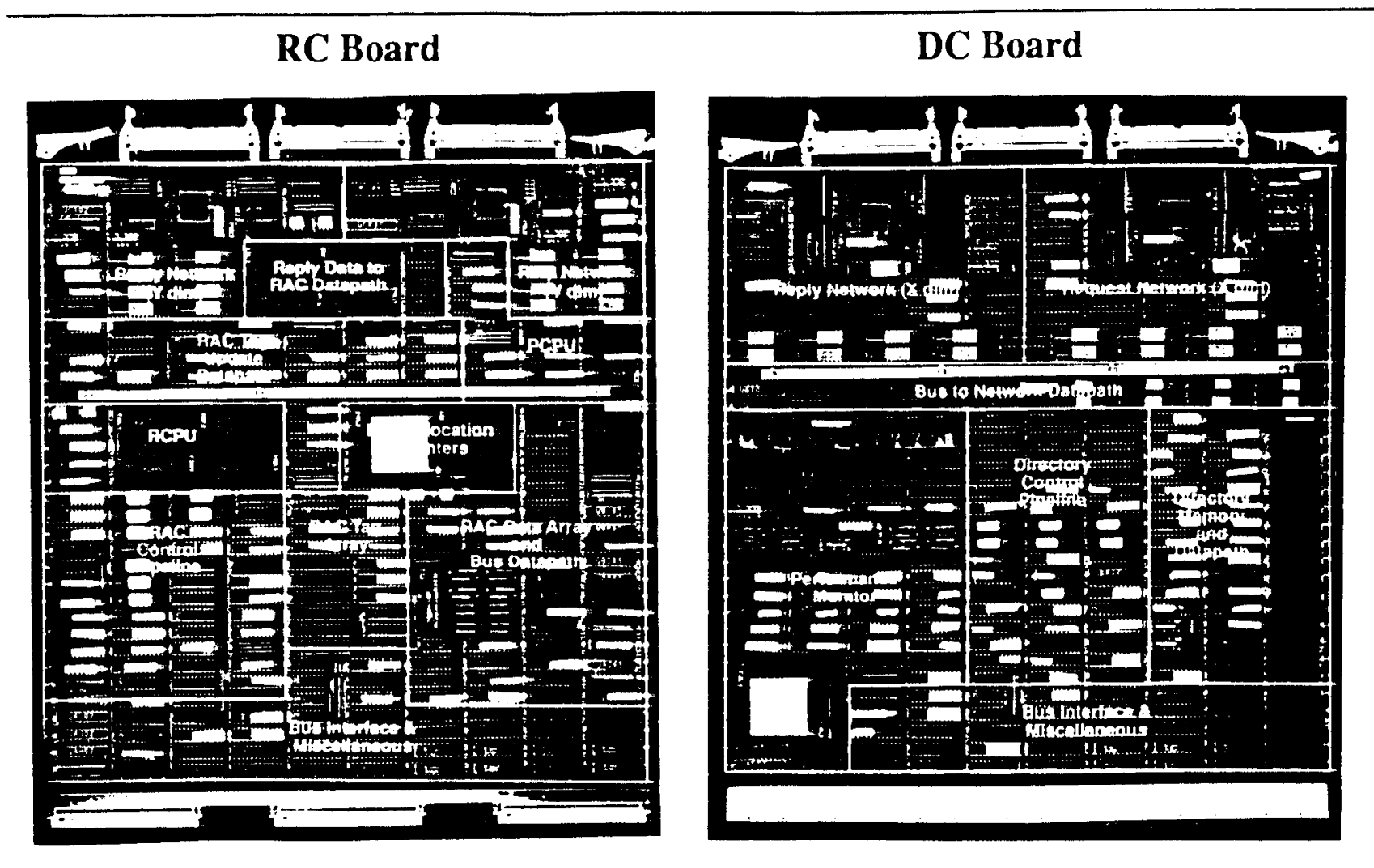

Figure 3. Directory and Reply Controller boards.

To use the $4 \mathrm{D} / 340$ in DASH, we have had to make minor modifications to the existing system boards and design a pair of new boards to support the directory memory and inter-cluster interface. The main modification to the existing boards is to add a bus retry signal that is used when a request requires service from a remote cluster. The central bus arbiter has also been modified to accept a mask from the directory which holds off a processor's recry until the remote request has been serviced. This effectively creates a split transaction bus protocol for requests requiring remote service. The new directory controller boards contain the directory memory, the intercluster coherence state machines and buffers, and a local section of the global interconnection network.

While the prototype, with minor modifications, could scale to support hundreds of processors, the current version is limited to a maximum configuration of 16 clusters and 64 processors. This limit was dictated primarily by the physical memory addressability of the 4D/340 system (256 Mbytes) which would severely limit the memory per processor in a larger system.

The directory logic in DASH is responsible for implementing the directory-based coherence protocol and interconnecting the clusters within the system. Pictures of the directory boards are shown in Figure 3. The directory logic is split between the two boards along the lines of the logic used for outbound and inbound portions of inter-cluster transactions.

The DC board contains three major subsections. The first section is the directory controller (DC) itself. which includes the directory memory associated with the cachable main memory contained within the cluster. The DC logic initiates all out-bound network requests and replies. The second section is the performance monitor which can count and trace a variety of intra- and inter-cluster events. The third major section is the request and reply outbound network logic together with the X-dimension of the nctwork itself.

The second board is the RC board which also contains three major sections. The first section is the reply controller (RC) which tracks outstanding requests made by the local processors and receives and buffers replies from remote clusters using the remote access cache (RAC). The second section is the pseudo-CPU (PCPU), which is responsible for buffering incoming requests and issuing these requests onto the cluster bus. The PCPU mimics a CPU on this bus on behalf of remote processors except that responses from the bus are sent out by the directory controller. The final section is the inbound network logic and the Y-dimension of the mesh routing networks.

Directory memory is accessed on each bus transaction. The directory information is combined with the type of bus operation, the address, and the result of snooping on the caches to determine what network messages and bus controls the $D C$ will generate. The directory memory organization is similar to the original directory scheme proposed by Censier and Feautrier [4]. Directory pointers are stored as a bit vector with 1 bit for each of the 16 clusters. While a full bit vector has limited scalability, it was chosen because it requires roughly the same amount of memory as a limited-pointer directory $[2,6,11,1]$ given the size of the prototype, and it allows for more direct measurements of the caching behavior of the machine. Each directory entry contains a single state bit that indicates whether the clusters have a shared or dirty copy of the data. The directory is implemented using DRAM technology, but performs all necessary actions within a single bus transaction.

The reply controller stores the state of on-going requests in the remote access cache (RAC). The RAC's primary rote is the coordi- 
nation of replies to inter-cluster transactions. This ranges from the simple buffering of reply data between the network and bus to the accumulation of invalidation acknowledgments and the enforcement of release consistency. The RAC is organized as a $128 \mathrm{Kbyte}$ direct-mapped snoopy cache with 16 byte cache lines. One port of the RAC services the in-bound reply network while the other snoops on bus transactions. The RAC is lockup-free in that it can handle several outstanding remote requests from cach of the local processors. RAC entries are allocated when a remote request is initiated by a local processor and persist until all inter-cluster transactions relative to that request have completed. The snoopy nature of the RAC naturally lends itself to merging requests made to the same cache block by different processors within the cluster, and it takes advantage of the cache-to-cache transfer protocol supported between the local processors. The snoopy stucture also allows the RAC to supplement the function of the processor caches. This includes support for a dirty-sharing state for a cluster (normally the Illinois protocol would force a write-back) and operations such as prefetch.

As stated in the architecture section, the DASH coherence protocol does not rely on a particular interconnection network topology. The prototype system uses a pair of wormhole routed meshes to implement the interconnection network. One mesh handles request messages while the other is dedicated to replies. The networks are based on variants of the mesh routing chips developed at Caltech where the datapaths have been extended from 8 to 16 bits $[5]$. Wormhole routing allows a cluster to forward a message after receiving only the first flit (flow unit) of the packet greatly reducing the latency through each node ( $\approx 50 \mathrm{~ns}$ per hop in our network). The bandwidth of each self-limed link is limited by the round trip delay of the request-acknowledge signals. In the prototype flits are transferred at approximately $30 \mathrm{MHz}$, resulting in a peak bandwidth of $120 \mathrm{Mbytes} / \mathrm{sec}$ in and out of each cluster.

\subsection{Gate Count Summary}

One important result of building the DASH prototype is that it provides a realistic model of the cost of directory-based cache coherence. While some of these costs are tied to the specific prototype implementacion (e.g., the full DRAM directory vector), they provide a complete picture of one system.

At a high level, the cost of the directory logic can be estimated by the fact that a DASH cluster includes six logic cards, four of which represent the base processing node and two of which are used for directory and inter-cluster coherence. This is a very conservative estimate, however, because Silicon Graphics' logic, in particular the MIPS processor chips and Silicon Graphics' gate arrays, are more highly integrated than the MSI PALs and LSI FPGAs used in the directory logic.

Table 1 summarizes the logic for a DASH cluster at a more detailed level. The table gives the percent of logic for each section and the totals in terms of thousands of 2 -input gates, kilobytes of static RAM, megabytes of dynamic RAM, and 16-pin IC equivalents. RAM bytes include all error detecting or correcting codes and cache tags. 16-pin IC equivalent is a measure of board area (0.36 sq. inch), assuming through-hole technology (i.e. DIPs and PGAs) was used throughout the design. (Actually about $1 / 4$ of the CPU logic is implemented in surface mount technology, but the IC Equivalent figures used here assume through-hole since all of the logic could have been designed in surface mount.) The number of 2 -input gates is an estimate based on the number of gate-array 2 . input gates needed to implement each function. For each type of logic used in the prototype the equivalent gate complexity was calculated as:

Custom VLSI Estimate based on part documentation.

CMOS Gate Array Actual gate count or estimate based on master-slice size and complexity.

PAL Translation of 2-level minimized logic into equivalent gates.

PROM Espresso minimized PROM files translated into 2-input gates. This includes the primary state machines in the DC and $R C$. but not the boot EPROMs for the CPUs.

TTL Gates in equivalent gate array macros.

Table 1. Percent of all logic in a DASH cluster.

\begin{tabular}{|l|c|c|c|c|}
\hline \multicolumn{1}{|c|}{ Section } & $\begin{array}{c}\text { Gates } \\
(\mathbf{0 0 0})\end{array}$ & $\begin{array}{c}\text { SRAM } \\
(\mathbf{K B})\end{array}$ & $\begin{array}{c}\text { DRAM } \\
(\mathbf{M B})\end{array}$ & $\begin{array}{c}\text { IC } \\
\text { Equiv }\end{array}$ \\
\hline \hline Processors/Caches & $69.3 \%$ & $86.0 \%$ & $0.0 \%$ & $32.4 \%$ \\
\hline Main Memory & $3.3 \%$ & $0.0 \%$ & $75.8 \%$ & $12.7 \%$ \\
\hline IO Board & $8.3 \%$ & $0.0 \%$ & $1.4 \%$ & $13.5 \%$ \\
\hline Directory Controller & $4.4 \%$ & $0.0 \%$ & $12.0 \%$ & $8.2 \%$ \\
\hline Reply Controller & $8.4 \%$ & $7.3 \%$ & $0.0 \%$ & $12.0 \%$ \\
\hline PCPU & $0.5 \%$ & $0.0 \%$ & $0.0 \%$ & $1.2 \%$ \\
\hline Network Outbound & $2.8 \%$ & $0.7 \%$ & $0.0 \%$ & $4.3 \%$ \\
\hline Network Inbound & $1.1 \%$ & $0.7 \%$ & $0.0 \%$ & $2.4 \%$ \\
\hline Performance Mon. & $1.8 \%$ & $5.3 \%$ & $10.8 \%$ & $3.5 \%$ \\
\hline Total & 535 & 2420 & 83 & 2603 \\
\hline
\end{tabular}

The numbers in Table 1 are somewhat distorted by the extra logic in the base Silicon Graphics' hardware and the directory boards that is not needed for normal operations. This includes (i) the performance monitor logic on the directory board: (ii) the diagnostic UARTs and timers attached to each processor; (iii) the Ethernet and VME bus interfaces on the Silicon Graphics' 1/O board. ${ }^{1}$ Table 2 shows the percentage of this core logic assuming the items mentioned above are removed.

As expected, only when measured in terms of IC equivalents (i.e. board arca), is the cost of the directory logic approximately $33 \%$. When measured in terms of logic gates the portion of the cluster dedicated to the directory is $20 \%$, and the SRAM and DRAM overhcad is $13.9 \%$ and $13.7 \%$ respectively.

Note that in the above analysis, we do not account for the hardware cost of snooping on the local bus separately because these costs are very small. In particular, the processor's two-level cache structure doesn't require duplicate snooping tags, and the processor's bus interface accounts for only $3.2 \%$ of the gates in a cluster. Even if the second-level cache tags were duplicated, it would represent only $4.0 \%$ of a cluster's SRAM. In practice, we expect most future systems will use microprocessors with integrated first-level caches (e.g. MIPS R4000, DEC Alpha, etc.) and to incorporate an external second-level cache (without duplicate tags) to improve uniprocessor perforpance. Thus, the extra SRAM cost for snooping (e.g., extra state bits) is expected to be negligible.

1. Each WO board would still include a SCSI interface for disk interfacing 
Table 2. Percent of core logic in a DASH cluster.

\begin{tabular}{|l|c|c|c|c|}
\hline \multicolumn{1}{|c|}{ Section } & $\begin{array}{c}\text { Gates } \\
(\mathbf{0 0 0})\end{array}$ & $\begin{array}{c}\text { SRAM } \\
(\mathrm{KB})\end{array}$ & $\begin{array}{c}\text { DRAM } \\
(\mathbf{M B})\end{array}$ & $\begin{array}{c}\text { IC } \\
\text { Equiv }\end{array}$ \\
\hline \hline Processors/Caches & $70.8 \%$ & $90.8 \%$ & $0.0 \%$ & $45.2 \%$ \\
\hline Main Memory & $3.9 \%$ & $0.0 \%$ & $86.3 \%$ & $13.9 \%$ \\
\hline IO Board & $5.1 \%$ & $0.0 \%$ & $0.0 \%$ & $10.3 \%$ \\
\hline Directory Controller & $5.2 \%$ & $0.0 \%$ & $13.7 \%$ & $9.0 \%$ \\
\hline Reply Controller & $9.9 \%$ & $7.7 \%$ & $0.0 \%$ & $13.1 \%$ \\
\hline PCPU & $0.6 \%$ & $0.0 \%$ & $0.0 \%$ & $1.3 \%$ \\
\hline Network Outbound & $3.3 \%$ & $0.8 \%$ & $0.0 \%$ & $4.7 \%$ \\
\hline Network Inbound & $1.3 \%$ & $0.8 \%$ & $0.0 \%$ & $2.6 \%$ \\
\hline Performance Mon. & $0.0 \%$ & $0.0 \%$ & $0.0 \%$ & $0.0 \%$ \\
\hline Total & 456 & 1268 & 73 & 2286 \\
\hline
\end{tabular}

Looking at the numbers in Table 2 in more detail aiso shows additional areas where the directory overhead might be improved. In particular, the prototype's simple bit vector directory grows in proportion to the number of clusters in the system, and in inverse proportion to cache line size. Thus, increasing cache line size from 16 to 32 or 64 bytes would reduce the directory DRAM overhead to $6.9 \%$ and $3.4 \%$ respectively, or it could allow the system to grow to 128 or 256 processors with the same $13.7 \%$ overhead. For larger systems, a more scalable directory structure $[2,6,11,1]$ could be used to keep the directory overhead at or below the level in the prototype. The directory's overhead in SRAM could also be improved. The $128 \mathrm{~KB}$ remote access cache (RAC) is the primary use of SRAM in the directory. The size of the RAC could be significantly reduced if the processor caches were lockup-free. With enhanced processor caches, the primary use of the RAC would be to collect invalidation acknowledgments and to receive granted locks. This would allow a reduction in size by at least a factor of four, and result in an SRAM overhead of less than 2\%. Likewise, a closer coupling of the base cluster logic and bus protocol to the inter-cluster protocol might reduce the directory logic overhead by as much as $25 \%$. Thus, the prototype represents a conservative estimate of directory overhead. A more ideal DASH system would have a logic overhead of $18-25 \%$, an SRAM overhead of $2-8 \%$ and a DRAM overhead $3-14 \%$. This is still significant, ${ }^{2}$ but when amortized over the cluster the overhead is reasonable.

The prototype logic distributions can also be extrapolated to consider other system organizations. For example, if the DASH cluster-based node was replaced by a uniprocessor node, the overhead for directory-based cache-coherence would be very high. Ignoring the potential growth in directory storage (that would need to track individual processor caches instead of clusters), the percent of directory logic in a uniprocessor node would grow to $\approx 44 \%$ (a 78\% overhead). Thus, a system based on uniprocessor nodes would lose almost a factor of two in cost/performance relative to a uniprocessor or small-scale multiprocessor.

Another possible system organization is one based on a general memory or messaging interconnech but without support for global hardware cache coherence (e.g., the BBN TC2000 or Intel Touchstone). An optimistic assumption for such a system is that it would remove all of the directory DRAM and support. the RAC and its datapath, and $90 \%$ of the RC and DC control pipelines. Under these assumptions, the fraction of logic dedicated to the inter-cluster interface falls to $10 \%$ of a cluster, and the memory overhead

2. Approximately equal to the complexity of a entire processor with its cactes. becomes negligible. Thus, the cost of adding inter-cluster coherence to a large-scale. non-cache coherent system is approximately $10 \%$. If more than a $10 \%$ performance gain is realized by this addition, then the overall cost/performance of the system will improve. Our measurements on DASH indicate that caching improves performance by far more than $10 \%$, and support for global cache coherence is well worth the extra cost.

Finally, by examining the required gate, memory, and connectivity requirements, one can estimate how the prototype logic might be integrated into a small number of VLSI components. As examined in detail in [9], such a system could consist of clusters based on the following:

- Four single-chip microprocessors with direct control of their second-level caches and a their interface to the cluster's snoopy bus.

- A single memory control chip interfaced to local DRAM and the cluster bus.

- An IO interface chip connecting the cluster to a high-speed fiber optic $1 / O$ links (e.g., fibre channel).

- A single directory controller chip interfacing to an external mixed SRAM-DRAM sparse directory, the cluster bus, and a network chip. ${ }^{3}$

- A single mesh routing chip supporting two logical 3-D meshes.

This integrated system could maintain a similar directory logic and memory overhead as the prototype, while supporting cache coherence for $2 \mathrm{~K}$ processors and $128 \mathrm{GBytes}$ of memory.

\subsection{Performance Monitor}

One of the prime motivations for building the DASH prototype was to study real applications with large data sets running on a large ensemble of processors. To enable more insight into the behavior of these applications when running on the prototype, we have dedicated over $20 \%$ of the DC board to a hardware performance monitor. Integration of the performance monitor with the base directory logic allows non-invasive measurements of the complete system without any external hardware. The performance hardware is controlled by software, and it provides low-level information on software reference characteristics and hardware resource utilizations. The monitor hardware can trace and count a variety of bus, directory and network events. The monitor is controlled by a software programmable Xilinx gate aray (FPGA)[15] allowing flexible event selection and sophisticated event prepro. cessing.

A block diagram of performance logic is shown in Figure 4. It consists of three major blocks. First, the FPGA which selects and preprocesses events to be measured and controis the rest of the performance logic. Second, two banks of $16 \mathrm{~K} \times 32$ SRAMs and increment logic that count event occurrences. Third, a $2 \mathrm{M} \times 36$ trace DRAM which captures 36 or 72 bits of information on each bus transaction.

The counting SRAMs together with the FPGA support a wide variety of event counting. The two banks of SRAM are addressed by events selected by the FPGA. They can be used together to trace events that occur on cycle by cycle basis, or the banks can be used independently to monitor twice as many events. By summing over all addresses with a particular address bit high or low the number of occurrences of that event can be determined. Likewise. the con-

3. A small associative RAC would be kept on-chip 


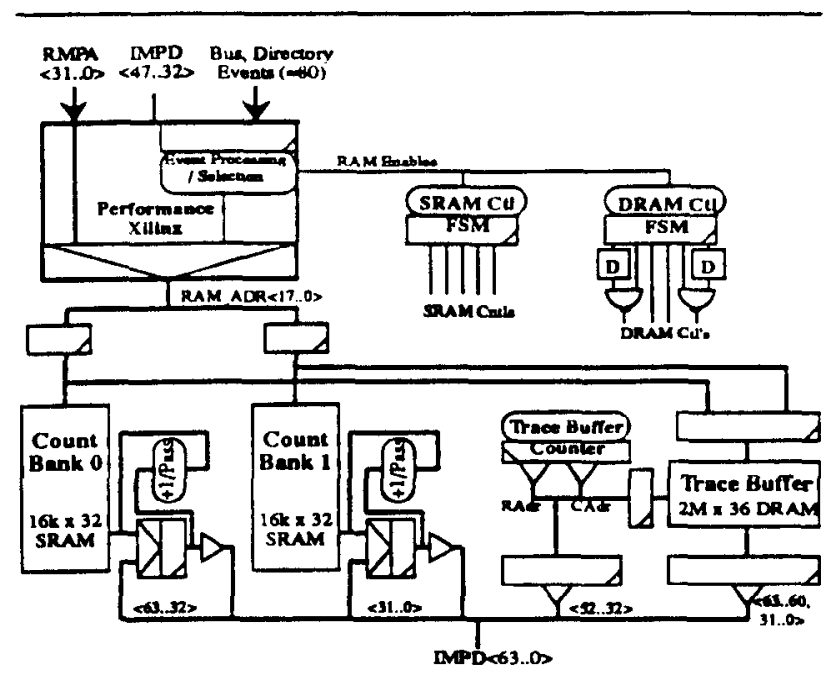

Figure 4. Block dlagram of the performance monitor logic

junction or disjunction of any set of events can be determined by summing over the appropriate address ranges. Another use of the count SRAM is as a histogram array. In this mode. certain events are used to start, stop and increment a counter inside the FPGA. The stop event also triggers the counter to be used as a SRAM address to increment.

The current use of the counting SRAM in the prototype increments the two banks of SRAM independently on each bus transaction. The data in the first bank allows access type frequencies, bus utilization, access locality, RAC performance, remote caching statistics and network message frequency to be calculated. The second bank of SRAM is addressed with the local cache snoop results and histogram counters of remote latency. The snoop data allows one to determine the effectiveness of cache-to-cache sharing within the cluster. The remote latency histogram dedicates an internal FPGA counter to each CPU which is enabled whenever a processor is waiting for a remote access. This allows a complete distribution of remote access latencies to be determined. Furthermore. when combined with the count of local bus cycles an estimase of processor utilization can be made.

The other resource of the performance monitor is a $2 \mathrm{M} \times 36$ trace array. Again. what information is traced can vary based on programming of the FPGA, but the current use of the trace logic has two modes. In the first configuration, up to $2 \mathrm{M}$ memory addresses together with the issuing processor number and read/write status are captured. The second mode can capture only $1 \mathrm{M}$ addresses, but adds additional bus and directory state, and a bus idle count to each trace entry. This trace information can be used to do detailed analysis of reference behavior or as input to a memory simulator. With software assistance the tracer can be used to capture much longer traces and trace all memory references.

\subsection{Prototype Performance}

This section examines the performance of the initial hardware prototype of DASH which includes 16 processors in four clusters. The first part summarizes the memory latencies measured on the prototype hardware. The second part describes the speedups obtained by parallel applications nun on the actual machine.

\subsection{Processor Issue Bandwidth and Latency}

Although the coherent caches in DASH significantly reduce the number of remote accesses made by a processor, it is still essential to minimize the latency when misses do occur. Table 3 lists the processor bandwidth and latency for cache memory operations in DASH assuming no contention. (PClocks refer to processor clocks which are $30 \mathrm{~ns}$ in the prototype.) The delays are based on measurements of the hardware, but extrapolated to a full $4 \times 4$ cluster configuration. In the table. the best-case numbers assume strideone access, with one cache miss every four references (cache lines are 16 bytes). The worst-case numbers assume stride-four accesses with no reuse of cache lines.

The table presents data separately for reads and writes. For reads, the access latency is given by the last column of the table. The latency can vary by more than two orders of magnitude depending on where a read access is serviced in the memory hierarchy. The read bandwidth also varies considerably, from a high of $133 \mathrm{Mbyt}$ es/sec from the primary cache to a meager 4 Mbytes/sec if all of the data is dirty in a remote non-home cluster. While, beyond a point, not much can be done about reducing the latency in largescale machines, the bandwidth can be increased via pipelining, and it is for this reason we have provided non-blocking prefetch operations in DASH. The times given for store operations are the rate at which writcs are retired from the write buffer into the second-level cache after acquiring ownership. Release consistency is assumed so that the processor need not wait for the write to retire, and invalidations do not affect write latency.

Table 3. Cache operation bandwidth and latencies.

\begin{tabular}{|l|c|c|c|c|}
\hline \multicolumn{1}{c|}{$\begin{array}{c}\text { Cache } \\
\text { Operation }\end{array}$} & \multicolumn{2}{c|}{ Best Case } & \multicolumn{2}{c|}{ Worst Case } \\
\cline { 2 - 5 } & $\begin{array}{c}\mathrm{MB} / \\
\text { sec }\end{array}$ & $\begin{array}{c}\text { Clock/ } \\
\text { word }\end{array}$ & $\begin{array}{c}\text { MB/ } \\
\text { sec }\end{array}$ & $\begin{array}{c}\text { Clock/ } \\
\text { word }\end{array}$ \\
\hline Read from 1st-level cache & 133.3 & 1.0 & 133.3 & 1.0 \\
\hline Fill from 2nd-lev. cache & 29.6 & 4.5 & 8.9 & 15.0 \\
\hline Fill from local bus & 16.7 & 8.0 & 4.6 & 29.0 \\
\hline Fill from remote & 5.1 & 26.0 & 1.3 & 101.0 \\
\hline Fill from dirty-remote & 4.0 & 33.8 & 1.0 & 132.0 \\
\hline Write retired in cache & 32.0 & 4.2 & 32.0 & 4.2 \\
\hline Write retired on local bus & 18.3 & 7.3 & 8.0 & 16.7 \\
\hline Write retired on remote & 5.3 & 25.3 & 1.5 & 88.7 \\
\hline Write retired on dirty-rem. & 4.0 & 33.0 & 1.1 & 119.7 \\
\hline
\end{tabular}

A more detailed break-down of the latency for local and remote cache misses is given in Figure 5. The latency for a local miss that is serviced within the cluster is based entirely on the base SGI hardware (i.e., the hardware we have added to the SGI clusters does not slow the system down). In the prototype, a simple remote miss (i.e., a miss that is serviced by a remote home cluster) takes $\approx 3.5$ times longer than a local miss. The final case illustrated in Figure 5 represents the latency for fetching a location that is dirty in a cluster other than its home. In this case, an extra 30 clocks (or $1 \mu \mathrm{sec})$ of delay is incurred in forwarding the request to the dirty cluster. The DASH protocol supports the direct transfer of the dirty data between the dirty and requesting cluster. reducing latency by $20 \%$ over a simpler protocol that first causes a writeback to the home cluster and then replies to the requesting processor.

While latencies in the DASH prototype (when measured in microseconds) are far from optimal, we believe that the delays when measured in processor clocks are quite indicative of what we expect to see in future large-scale machines. The reason is that 


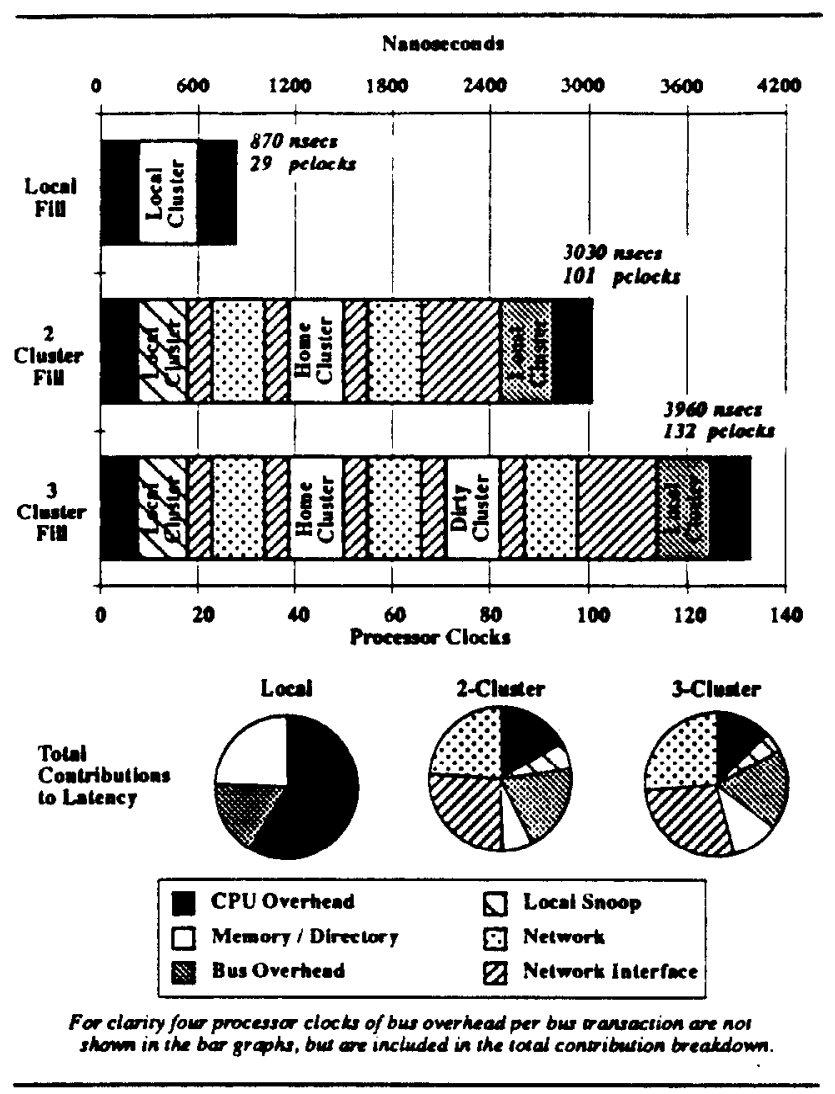

Figure 5. Cache fill latency in the DASH prototype.

while state-of-the-art technology (with integration and optimization) would allow us to reduce the prototype's latencies by a factor of about three [9], state-of-the-art processor clock rates are also about three times the $33 \mathrm{MHz}$ used in the prototype. As a result, we expect that exploiting cache and memory locality will continue to be imporant in future large-scale machines, as will mechanisms that help hide or tolerate latency.

\subsection{Parallel Application Performance}

This subsection outlines the performance actually achieved on the prototype for a number of parallel applications. We begin by describing the software environment available on the prototype and how the measurements were made. We then present the speedup for nine parailel programs representing a variety of application domains. Three of these applications are studied more in greater detail using data captured by the performance monitor.

\subsubsection{Application Runtime Environment}

The operating system running on the prototype DASH is a modified version of IRLX: a variant of UNIX System V.3 developed by Silicon Graphics. The applications for which we present results are coded in $C$ that has been augmented with the Argonne National Labs (ANL) parallel macros [10] to control a MLMD, shared-memory programming model.

Before giving the speedup results in the next subsection, we first state the assumptions used in measuring the speedups. The speedup were measured as the time for the uniprocessor to execute the parallel version of the application code (i.e. not all synchronization code is removed) divided by the time for the parallel appli- cation to run on a given number of processors. The runs were averaged over a number of executions to eliminate any scheduling anomalies in UNTX. In some applications, the serial start-up time is ignored from the measurement because the runs used shortened executions of the application (less time steps, etc.) to reduce measurement time. In production runs the start-up time would be negligible.

For our measurements, each application process is attached to a processor for its lifetime, and we fully use one cluster before assigning processors to new clusters. Physical memory pages used by the application are allocated oniy from the clusters that are actively being used. as long as the physical memory in those clusters is enough. Thus, for an application running with 4 processes, all memory is allocated from the local cluster, and all misses cost about 30 clocks. However, with 8 processes, some misses may be to a remote cluster and cost over 100 clock cycles. Most of the programs allocate shared data randomly or in a round-robin fashion from the clusters being actively used, but some include explicit system calls to control memory allocation. ${ }^{4}$ Finally, all applications were run under processor consistency mode, i.e., writes were not retired from the write-buffer until all invalidation acknowledgments had been received, and no prefetching has been added.

\subsubsection{Application Speedups}

Figure 6 gives the speedup for nine parallel applications running on the hardware prototype using from 1 to 16 processors. The applications cover a variety of domains. There are some scientific applications (Bames-Hut and Water), several engineering applications (Radiosity, MP3D, PSIM4, Locusroute) and two kernels (Cholesky, Matrix Multiply and Mincut). Four of the programs (Water. LocusRoute, MP3D and Cholesky) are taken from the SPLASH parallel application suite[14]. We begin with a quick overview of all nine applications and then present the detailed reference behavior and performance of three of the applications (Barnes-Hut, Water, and LocusRoute).

Starting with the applications with the best speedup. the Radiosity application is from the domain of computer graphics. It computes the global illumination in a room given a set of surface patches and light sources using a variation of hierarchical $n$-body techniques. The particular problem instance solved starts with 364 surface patches, and ends with over 10,000 patches. While the data structures used are a complex oct-tree and a binary-space partition tree, we see that caches work quite well and we get a speedup of over 14 with 16 processors. The second application. Bames-Hut, is an $\mathbf{N}$-body galactic simulation solved using the Bames-Hut algorithm (an $O(M \log N)$ algorithm). Again we see that although the structure of the program is complex, good speedups are obtained.

The next application is Mincut. It performs graph partitioning using parallel simulated annealing. We ran Mincut to find the minimum bisection of a graph containing 500 nodes. Mincul achieves good speedups because there is extensive cache reuse as processors traverse the graph and repeatedly consider moving nodes as the graph partition and annealing temperature change.

The next application is a scaied matrix multiply. It uses $88 \times 88$ square matrices on a single processor (obtaining 8.8 DP MFLOPS on a single processor) and $1408 \times 1408$ matrices on 16 processors (obtaining 125 DP MFLOPS). The application below that is the Water code (the parallelized version of the MDG code from the

\footnotetext{
4. Currently all openting system code and data are allocated from cluster-0's mem ory. This caures cluster $D$ to become ho spo for OS missea, and causea some degradation in speedups. We are in the process of fixing this problem.
} 


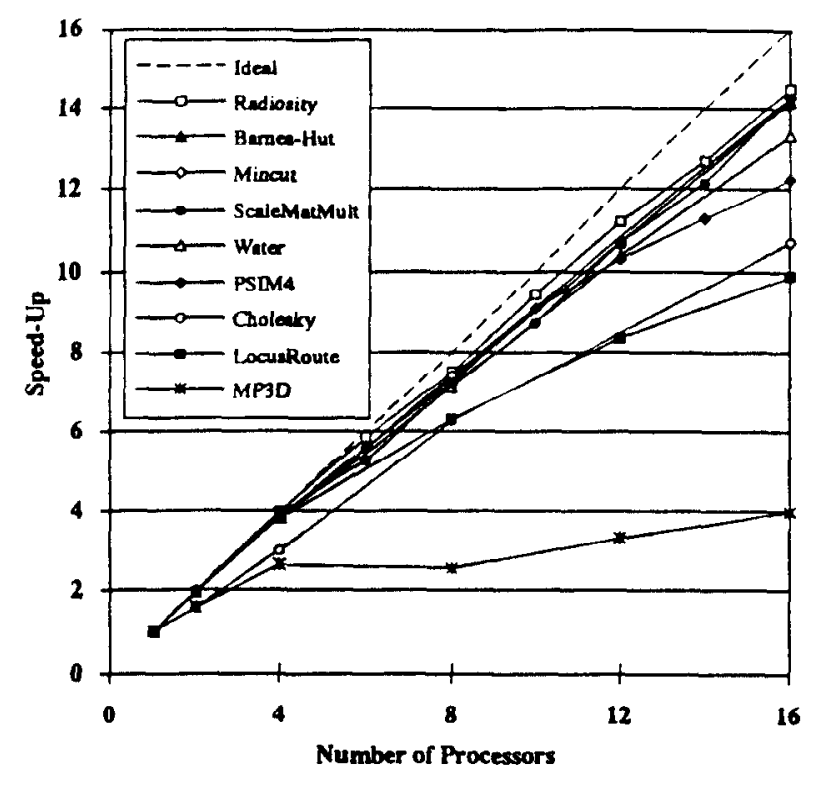

Figure 6. Speedup of applications on the DASH prototype.

Perfect Club benchmarks). a molecular dynamics code. We measured runs using 512 water molecules.

PSIM4 is an application from NASA-Ames, that is a particlebased simulator of a wind tunnel. PSIM4 is an enhanced version of the MP3D code (the application with the poorest speedup), both in terms of functionality (it models multiple types of gases and it models chemistry) and in terms of locality of memory accesses (it uses spatial decomposition of simulated space to distribute work among processors). The 16 processor nun is done with over 100,000 particles and it achieves a scaled speedup of over 12 in contrast to a speedup of about 4 achieved by the older MP3D code. While the changes in functionality make a direct comparison of absolute execution time for PSIM4 and MP3D impossible, the speedup improvements of PSIM4 over MP3D are very encouraging.

The next application is Cholesky. It performs factorization of sparse positive-definite matrices using supernodal techriques (data blocking techniques that enhance the performance of caches both for uniprocessors and multiprocessors). Here it is used to solve a $256 \times 256$ grid problem. We note that much of the fall off in speedup that we see is due to the trade-off between large data block sizes (which increase processor efficiency, but decrease available concurrency and cause load balancing problems) and small data block sizes. As we go to a large number of processors. we are forced to use smaller block sizes unless the problem size is scaled to unreasonably large sizes. The next application is LocusRoute which performs global routing of standard cells. It will be discussed later in this section.

Finally, as stated before. MP3D is a particle-based wind-tunnel simulator. The measured runs simulated 40,000 particles. The speedups for MP3D are poor because the particles are statically allocated to processors, but the space cells (representing physical space in the wind turnel) are referenced in a relatively random manner depending on the location of the particie being moved. Since each move operation also updates the corresponding space cell, as the number of processors increases, it becomes more and more likely that the space cell being referenced will be dirty in another processor's cache. Thus. even with four processors, the speedup is poor. When a second cluster is added, speedup is flat because roughly half of the misses are now remote. MP3D's speedups improve for 12 and 16 processors, but this does not compensate for the initial inefficiencies encountered with 4 and 8 processors.

Overall, we see that many applications achieve good speedups. even though they have not been specially optimized on the DASH prototype. Almost all get over ten times improvement on sixteen processors, and some get above fourteen times speedup.

\subsubsection{Detailed Case Studies}

To get a better understanding of the detailed reference behavior of these applications, we now examine Barnes-Hut. Water, and LocusRoute in more detail. We also extend the results for these applications with preliminary results from the 32 -processor DASH system (which has just been up for the last two weeks). As expected from Figure6, Barnes-Hut and Water achieve good speedups on 32 processors (27.5 and 24.5 respectively), but the speedups for LocusRoute fall-off significantly $(9.9$ at 16 , but only 13.2 at 32). These applications were chosen because they achieved a range of speedups, and we were able to get results for them on the larger 32-processor system. We expect that once the 32-processor kemel is better tuned the speedups will improve.

\subsubsection{Bames-Hut}

The Barnes-Hut program [13] models the dynamic evolution of a system of galaxies under gravitational forces. Using the BamesHut algorithm. the possible $N^{2}$ interactions are reduced to $M \log N$ by a hierarchical decomposition of the galaxies and by approximating groups of distant bodies by a single point at their center of mass. The input to the measured runs consisted of two interacting Plummer-model galaxies with 16384 bodies each.

Table 4 gives a detailed memory reference profile of Barnes-Hut ruming on DASH as measured by the hardware performance monitor. ${ }^{5}$ Table 5 is broken into four sections: (i) overall performance and processor utilizarion; (ii) memory request distribution; (iii) request locality and latency and (iv) bus and network utilization.

The first section of the table gives the overall speedup, efficiency relative to the uniprocessor, and processor utilization. Unfortunately, processor utilization cannot be measured directly from the bus, so the number in the table is an estimate that assumes the processor is doing active work whenever it is not waiting for a bus transaction to complete. This ignores internal stalls due to firstlevel cache misses satisfied by the second-level, TLB miss handling, and floating-point interlocks. ${ }^{6}$ The number of busy clocks between stalls (hird row) give an indication of cache hit rate and the application's sensitivity to memory latency.

As indicated by Table 4, Barnes-Hut has a high and nearly constant cache-hit ratio, and a large fraction of its misses are local. Thus, processor efficiency and speedup are very good as the number of

5. The resulus given in the table are averaged over all active clusters. This implies come inaccuracies for the one and two processor nuns due wo the overtead of the idle processors running the UNDX scheduler and dacenons (e.g., slightly lower proceseor utilization for 1 and 2 processon than for 4 processors).

6. Since writes are buffered, they are not sssumed to stall the processor directly. Instead, it is assumed that the processor can execule for 20 clocks before stalling. This delay is an estimate of the time for the processor wo fill the other 3 words wo the write-buffer (i.e. assuming $15 \%$ instructions are writes). In reality, the processor may not issue writes at this rate, or may stall earlier due to a first-level cache mise. 
processors increases. There is a small drop in efficiency as the first remote cluster is added, but the degradation beyond this is slight.

The second section of Table 4 gives a breakdown of the memory reference types. This breakdown indicates the type of accesses that cause bus transactions and whether synchronization references are significant. In Bames-Hut, cache read misses dominate and thete are few synchronizations.

The third section of Table 5 lists the fraction of local cache fills, the fraction of remote fills satisfied by a dirty-remote cluster (ratio of 3 cluster to 2 cluster fills), and the latency for local and remote cache fills. The locality figure counts any references satisfied in a single bus transaction as local, while any that must be repeated are considered remote. Thus, a remote reference that was satisfied by a local cache-to-cache transfer between processors would be considered local, and a local reference that was dirty-remote would be considered remote. ${ }^{7}$

For Bames-Hut, cluster locality is high and decreases only slightly as clusters are added. The actual locality of references (not given in the table) does decrease as clusters are added, but most communication is with nearby processors. Thus, even though the home for the data is remote, many remote accesses are satisfied by a local cache-to-cache transfer from another processor in the cluster. In addition, of the references that are remote, most misses are serviced by the home cluster. This results from the fact that most of these misses are to global data, and only the first processor needs to fetch this data from the producing (i.e. dirty) cluster. The others read the shared data from the home.

The final section of Table 4 indicates the load on the cluster bus and on the bisection of the mesh networks. Bus utilization is measured directly by the performance monitor, while the network bisection utilizations are estimates assuming uniform network traffic. The bisection utilizations are calculated by knowing the total number of network messages sent. assuming half of the messages cross the bisection of the mesh, and dividing by the bandwidth provided across the bisection. ${ }^{8}$ For Bames-Hut, both the buses and networks are lightly loaded.

In Barnes-Hut, as in the other applications studied, the bus loading is higher than the network bisection loading. This is due to the relatively slow cluster bus used in the prototype, and the retry mechanism used for the remote references which implies that each remote reference includes at least three bus accesses, but only one network request and one network reply. For the size of the prototype, the individual cluster buses limit total memory bandwidth. In larger systems with more nodes and faster split-transaction cluster buses, we expect that the network bisection will limit aggregate memory bandwidth if accesses are uniformly distributed.

\subsubsection{Water}

Water is a molecular dynamics code from the field of computational chernistry. The application computes the inter action between a set of water molecules over a series of time steps. The algorithm is $O\left(N^{2}\right)$ in that each molecule interacts with all other molecules in

\footnotetext{
7. The 20-25\% dirty-renote fills when using 1-4 processers are back ground OS activity. This is onily $20-25 \%$ of the $0.5-3.0 \%$ of the misses that are remore. Local mises caused by the applicstion dominace. When using 8 processors, the dirtyremoxe pereentages are low because there are never any three ciuster fills because there is only two active clusters.

8. The factor of two increase when going from 12 to 16 processors arises because the 32-processor DASH is arranged in $14 \times 2$ grid. Currently, processors are numbered and allocated first in the $X$-dimension so that the 16 processors run in $a 4 x 1$ ensemble. Thus, a single $X$-dimension path sees the load of all four clusters.
}

the system. As shown in Figure 6, the Water application achieves good speedup on the DASH hardware.

Table 5 shows that cache-locality is high in Water, and the time between processor stalls indicates that it is not highly sensitive to memory latency. The table does show some reduction in the busy clocks between stalls, especiaily when going from 2 to 4 processors. but the decrease is slight after this initial drop. In comparison with Bames-Hut. Water achieves a slightly lower speedup due to lower cluster locality which increases the average miss penalty. As indicated by the fraction of dirty-remote cache reads, many more of the misses in Water are satisfied directly by the producing processor than in Bames-Hut.

Looking at the breakdown of memory reference types in Water, the percentage of synchronization references is fairly high. This is due in part to the high cache hit rates, and to the fact that every successful lock acquire or release references the bus in the prototype. Given the percentage of locks and unlocks are almost identical, this data also indicates that lock contention is not a problem in Water.

Finally, we see that like Bames-Hut, Water does not put a heavy load on the memory system. While the table does indicate an increase in remote memory latency, this is due to the increasing fraction of remote misses that are dirty-remote as opposed to larger queuing delays.

\subsubsection{LocusRoute}

LocusRoute is a standard-cell placement tool that uses actual routed area to evaluate the quaiity of a given placement. Thus. the task that LocusRoute performs is the routing of a given cell placement. LocusRoute exploits parallelism at two levels. First, multiple wires are routed simultancously. Second. different routes for the same wire are evaluated in parallel. The runs shown in Figure 6 were for the route of a circuit consisting of 3817 wires and 20 routing channels.

LocusRoute achieves a respectable 9.9 times speedup on 16 processors, but speedup does not increase linearly when more than 16 processors are used (onily 13.2 times speedup on 32 processors). Comparing the reference behavior of LocusRoute shown in Table 6 with that of Water, it is clear why LocusRoute does not achieve the same speedup. First, its cache hit rate (busy pclocks between stalls) is lower than Water. This makes it more sensitive to the increase in memory latency when going to multiple clusters. Second, as in Water, locality falls off with more processors and the fraction of dirty-remote references also increases.

Considering the application data structures and algorithms, these effects are not surprising. In LocusRoute, most misses are due to the cost array which tracks the number of signals routed in a given section of a channel. The cost array is actively updared by each processor, and there is only limited reuse of the data structure as wires are routed. The result is a lower hit rate than Water and more sensitivity to locality.

Looking at system loading, it is clear that LocusRoute puts more stress on the memory system than Barnes-Hut or Water. Bus utilization is moderate $(35-40 \%)$. The large latencies for remote memory references when using 24 or 32 processors are due in part to hot spotting for the cost array. In the current implementation, this array is allocated only out of cluster 0's memory. For the 32-processor run, this cluster has over $65 \%$ bus utilization (as compared to the average of $37 \%$ ), and we suspect there is substantial queuing at the PCPU. While removing this hot spot should improve performance, we expect that LocusRoute will achieve significanty better speedup on more than 16 processors only with larger problems. 
Table 4. Barnes-Hut memory access characterístics

\begin{tabular}{|l|r|r|r|r|r|r|r|r|}
\hline \multicolumn{1}{|c|}{ Execution Attribute } & 1 Proc. & 2 Proc. & 4 Proc. & 8 Proc. & 12 Proc. & 16 Proc. & 24 Proc. & 32 Proc. \\
\hline \hline Speedup & 1.0 & 2.0 & 3.9 & 7.4 & 10.7 & 14.2 & 20.6 & 27.5 \\
\hline Efficiency (relative to uniprocessor) & 1.00 & 0.99 & 0.96 & 0.92 & 0.89 & 0.89 & 0.86 & 0.86 \\
\hline Busy Pclks between Proc. Stalls & 526.3 & 633.8 & 602.7 & 606.8 & 534.6 & 602.7 & 564.5 & 560.9 \\
\hline Est. Processor Utilization (\%) & 94.6 & 95.6 & 95.5 & 94.2 & 92.9 & 93.4 & 92.5 & 92.3 \\
\hline Cache Read (\%) & 85.6 & 90.3 & 88.5 & 91.5 & 92.0 & 90.7 & 90.5 & 90.4 \\
\hline Cache Read Exclusive (\%) & 9.7 & 6.8 & 10.1 & 6.5 & 6.1 & 6.9 & 6.7 & 6.7 \\
\hline Cache Lock (\%) & 2.3 & 1.4 & 0.7 & 1.1 & 1.2 & 1.6 & 2.0 & 2.0 \\
\hline Cache Undock (\%) & 2.1 & 1.3 & 0.7 & 0.8 & 0.7 & 0.8 & 0.6 & 0.6 \\
\hline Fraction of Reads Local (\%) & 97.8 & 99.0 & 99.5 & 89.8 & 86.8 & 84.9 & 82.6 & 82.5 \\
\hline Fraction of Rem. Rds. Dirty-Rem (\%) & 21.8 & 21.0 & 21.2 & 9.1 & 6.4 & 5.7 & 5.5 & 5.3 \\
\hline Avg Local Cache Fill (Pclks) & 29.2 & 29.2 & 29.3 & 29.4 & 29.4 & 29.4 & 29.4 & 29.4 \\
\hline Avg Rem Cache Fill (Pclks) & 106.6 & 107.4 & 105.7 & 104.2 & 106.2 & 109.1 & 110.9 & 113.1 \\
\hline Bus Utilication (\%) & 5.2 & 6.3 & 9.1 & 9.8 & 11.0 & 10.2 & 10.9 & 11.0 \\
\hline Req. Net Bisection Util. (\%) & 0.6 & 0.7 & 0.7 & 0.8 & 0.9 & 1.7 & 1.9 & 2.0 \\
\hline Reply Net Bisection Util. (\%) & 0.5 & 0.5 & 0.5 & 0.9 & 1.1 & 2.2 & 2.5 & 2.6 \\
\hline
\end{tabular}

Table 5. Water memory access characteristics

\begin{tabular}{|c|c|c|c|c|c|c|c|c|}
\hline Execution Attribute & 1 Proc. & 2 Proc. & 4 Proc. & 8 Proc. & 12 Proc. & 16 Proc. & 24 Proc. & 32 Proc. \\
\hline Speedup & 1.0 & 2.0 & $=3.8$ & 7.1 & 10.4 & 13.3 & $=19.3$ & 24.6 \\
\hline Efficiency (relative to uniprocessor) & 1.00 & 0.99 & 0.95 & 0.89 & 0.87 & 0.83 & 0.80 & 0.77 \\
\hline Busy Pclks between Proc. Stalls & 935.9 & 955.0 & 614.6 & 528.3 & 515.0 & 523.7 & 553.8 & 506.3 \\
\hline Est. Processor Utilization (\%) & 97.5 & 97.7 & 97.2 & 93.2 & 91.1 & 90.6 & 89.2 & 88.0 \\
\hline Cache Read $(\%)$ & 46.0 & 45.2 & 46.7 & 46.9 & 45.2 & 45.2 & 44.6 & 47.0 \\
\hline Cache Read Exclusive (\%) & 11.9 & 7.3 & 25.1 & 29.6 & 30.9 & 30.9 & 31.7 & 30.8 \\
\hline Cache Lock (\%) & 20.9 & 23.8 & 14.1 & 12.1 & 12.4 & 12.5 & 12.3 & 11.8 \\
\hline Cache Unlock (\%) & 20.8 & 23.7 & 14.1 & 11.4 & 11.6 & 11.4 & $\overline{11.5}$ & 10.5 \\
\hline Fraction of Reads Local (\%) & 96.9 & 97.2 & 99.4 & 68.9 & 55.1 & 51.6 & 40.9 & 42.8 \\
\hline Fraction of Rem. Rds. Dirty-Rem (\%) & 21.4 & 21.6 & 22.6 & 9.2 & 18.3 & 27.0 & 48.0 & 50.0 \\
\hline Avg Local Cache Fill (Pclks) & 29.2 & 29.2 & $\overline{29.4}$ & 29.6 & 29.7 & 29.7 & 29.7 & 29.8 \\
\hline Avg Rem Cache Fill (Pclks) & 102.8 & 107.5 & 106.9 & 104.5 & 108.1 & 111.6 & 118.3 & 120.6 \\
\hline Bus Utilization (\%) & 4.6 & 5.7 & 10.2 & 14.5 & 16.3 & 16.6 & 17.7 & 18.8 \\
\hline Req. Net Bisection Util. (\%) & $\overline{0.7}$ & $\overline{0.8}$ & 0.7 & 1.5 & 2.0 & 4.6 & 6.1 & 6.7 \\
\hline Reply Net Bisection Util. (\%) & 0.5 & 0.6 & 0.5 & 1.8 & 2.5 & 5.3 & 6.3 & 6.7 \\
\hline
\end{tabular}

Table 6. LocusRoute memory access characteristics

\begin{tabular}{|l|r|r|r|r|r|r|r|r|}
\hline \multicolumn{1}{|c|}{ Execution Attribute } & 1 Proc. & 2 Proc. & 4 Proc. & 8 Proc. & 12 Proc. & 16 Proc. & 24 Proc. & 32 Proc. \\
\hline \hline Speedup & 1.0 & 2.0 & 3.8 & 6.3 & 8.4 & 9.9 & 11.9 & 13.2 \\
\hline Efficiency (relative to uniprocessor) & 1.00 & 0.99 & 0.95 & 0.79 & 0.70 & 0.62 & 0.50 & 0.41 \\
\hline Busy Pclks between Proc. Stalls & 342.8 & 312.4 & 258.0 & 196.1 & 179.4 & 175.4 & 179.9 & 181.2 \\
\hline Est. Processor Utilization (\%) & 92.7 & 92.9 & 92.0 & 79.1 & 73.1 & 69.5 & 64.8 & 59.4 \\
\hline Cache Read (\%) & 74.0 & 70.3 & 68.8 & 64.5 & 69.0 & 69.3 & 69.8 & 70.1 \\
\hline Cache Read Exclusive (\%) & 15.8 & 21.9 & 25.8 & 31.3 & 27.5 & 27.2 & 26.5 & 26.0 \\
\hline Cache Lock (\%) & 5.0 & 3.9 & 2.7 & 2.3 & 2.0 & 2.1 & 2.4 & 2.7 \\
\hline Cache Unlock (\%) & 5.0 & 3.8 & 2.6 & 2.0 & 1.5 & 1.4 & 1.3 & 1.0 \\
\hline Fraction of Reads Local (\%) & 97.1 & 98.4 & 99.6 & 63.7 & 60.7 & 54.4 & 46.5 & 41.3 \\
\hline Fraction of Rem. Rds. Dirty-Rem (\%) & 24.9 & 24.7 & 22.8 & 2.9 & 20.8 & 28.0 & 33.6 & 35.0 \\
\hline Avg Local Cache Fill (Pclks) & 29.1 & 29.3 & 29.7 & 30.4 & 30.8 & 31.0 & 31.0 & 30.9 \\
\hline Avg Rem Cache Fill (Pclks) & 108.2 & 106.9 & 107.3 & 109.1 & 119.6 & 128.7 & 149.6 & 183.0 \\
\hline Bus Utilization (\%) & 5.9 & 8.9 & 17.1 & 30.8 & 36.1 & 38.2 & 38.5 & 36.8 \\
\hline Req. Net Bisection Util. (\%) & 0.6 & 0.6 & 0.7 & 3.8 & 5.9 & 14.2 & 16.1 & 16.1 \\
\hline Reply Net Bisection Util. (\%) & 0.5 & 0.5 & 0.5 & 4.9 & 6.7 & 15.4 & 16.8 & 16.6 \\
\hline
\end{tabular}




\subsubsection{Application Speedup Summary}

Overall, a number of conclusions can be drawn from the speedup and reference statistics presented in previous sections. First, it is possible to get near linear speedup on DASH for a number of real applications. Applications with the best speedup have good cache and cluster locality. Since most of the degradation in memory latency occurs when adding the first remote cluster, we expect most of the applications will perform well on 32 and 64 processors. Speedup for many of the applications should be more than 24 on 32 processors, and more than 45 on 64 processors, especially if problem size is increased.

In absolute terms, the number of busy clocks between bus accesses indicates that caching of shared data improves performance significantly. For example, earlier simulation work with the SPLASH benchmarks [14] indicates that the reference rates for Water and LocusRoute to shared data (with 32 processors) is roughly one reference every 20 and 11 instructions respectively. Given the number of busy clocks between misses for Water and LocusRoute given in Table 5 and Table 6 (or even assuming that the number of processor instructions between stalls is optimistic by a factor of two due to internal stalls), caches are satisfying $92 \%$ of the shared references in Water (i.e. there are at least $506 / 2 / 20 \approx 12$ shared references for every miss), and $88 \%$ of the shared references in LocusRoute. Thus, processor utilization without caching would be only $26 \%$ in Water and only $13 \%$ in LocusRoute. ${ }^{9}$ Overall, this implies that caching of shared data improves performance by a factor of 3.4-4.5 in these applications, but as shown earlier, only adds $10 \%$ to system cost. 10

Even with caches, however, locality is still important. If locality is very low and communication misses are frequent (as in MP3D), then speedup will be poor. However, for many applications, the natural locality of the applications is enough (e.g., Bames-Hut, Radiosity. Water) that good speedups can be achieved without algorithmic or programming contortions. Even in applications where natural locality is limited. DASH's shared-address space model allows the programmer to focus on the few critical data structures that are causing loss in performance, rather than having to explicitly manage (replicate and place) all data objects in the program.

\subsection{Conclusions}

This paper has outlined our experience in building and staring to use the DASH prototype system. The first result from building the prototype is that such systems are feasible. While the coherence protocol and hardware are not trivial, such systems can be built. Looking in more detail at the logic and memory costs exhibited by the prototype, we have shown that the logic overhead for supporting distributed shared memory (without coherence) is about $10 \%$. Supporting cache coherence adds another $10 \%-14 \%$ in logic and memory overhead if clustering is used.

The second result of building the prototype has been an analysis of the memory system performance. At the lowest level, it is clear that remote memory latencies are significant. We believe this will remain true as processor speeds increase relative to the inherent delays of a large system. Thus, both cache and cluster locality are

9. This assumes the locality of shared references is the same as the locality given in the table. This is optimistic since there would be no cache-lo-cache shering.

10. The extra overhead costs for caching is conservalive because we ignore any added costs in the uncachod system that would be necessary to increase the band. widih to main menury. important in this class of machines, and latency hiding techniques (e.g.. prefetch) may be very useful.

The prototype system has also allowed us to measure applications with large data sets using the performance monitor hardware. A number of parallel applications have been run and most achicve good speedup. Many of these applications achieve better than 12 times speedup on 16 processors, and the preliminary results with the 32-processor machine indicate that many will also work weil with 32 and 64 processors.

\section{Acknowledgments}

This research has been supported by DARPA contract N00039-91 C-0138. Dan Lenoski is supported by Tandem Computers Inc. James Laudon is supported by IBM. Anoop Gupta is partly supported by a NSF Presidential Young Investigator Award.

\section{References}

[1] Agarwal, A., B.-H. Lim, D. Kranz, and J. Kubiatowicz. LimitLESS Directories: A Scalable Cache Coherence Scheme. In Proc. Fourth Int. Conf. on Architectural Support Programming Languages and Operating Systems. pp. 224234, 1991.

[2] Agarwal, A., R. Simoni, J. Hennessy, and M. Horowitz. An Evaluation of Directory Schemes for Cache Coherence. In Proc. 15th Int. Symp. on Computer Architecture. pp. 280289, 1988.

[3] Basketh F., T. Jermoluk, and D. Solomon. The 4D-MP Graphics Superworkstation: Computing + Graphics $=40$ MIPS + 40 MFLOPS and 100,000 Lighted Polygons per Second. In Proc. Compcon Spring 88. pp. 468-471, 1988.

[4] Censier, L. and P. Feautrier, A New Solution to Coherence Problems in Multicache Systems. IEEE Trans. on Computers, C(27):1112-1118, 1978.

[5] Flaig, C.M., VLSI Mesh Routing Systems. Technical Report 5241:TR:87, Califomia Institute of Technology, May 1987.

[6] Gupta, A., W.-D. Weber, and T. Mowry. Reducing Memory and Traffic Requirements for Scalable Directory-Based Cache Coherence Schemes. In Proc. 1990 Int. Conf. on Parallel Processing. pp. I:312-321, 1990.

[7] Lenoski, D., J. Laudon, K. Gharachorloo, A. Gupta, and J. Hennessy. The Directory-Based Cache Coherence Protocol for the DASH Multiprocessor. In Proc. 17th Int. Symp. on Computer Architecture. pp. 148-159, 1990.

[8] Lenoski, D. J. Laudon, K. Gharachorloo, W.-D. Weber, A. Gupta, J. Hennessy, M. Horowitz, and M. Lam. The Stanford DASH Multiprocessor. Computer, 25(3), 1992.

[9] Lenoski, D.E., The Design and Analysis of DASH: A Scalable Directory-Based Multiprocessor. Ph.D. Thesis. Stanford University. 1991. Also available as Stanford University Technical Report CSL-TR-92-507

[10] Lusk, E., R. Overbeek, J. Boyle, R. Butler, T. Disz, B. Glickfeld, J. Patterson, and R. Stevens, Portable Programs for Parallel Processors. Holt, Rinehard and Winston. Inc. 1987. 
[11] O'Krafka. B.W, and A.R. Newton. An Empirical Evaluation of Two Memory-Efficient Directory Methods. In Proc. 17th Int. Symp. on Computer Archisecture. pp. 138-147, 1990.

[12] Papamarcos. M.S. and J.H. Patel. A Low Overhead Coherence Solution for Multiprocessors with Private Cache Memories. In Proc. 11 th Int. Symp. on Compuser Architecture. pp. 348-354, 1984.

[13] Singh, J.P., C. Holt, T. Totsuka, A. Gupta, and J.L. Hennessy, Load Balancing and Data Locality in Parallel $N$-body Techniques. Technical Report CSL-TR-92-505. Stanford University, 1991

[14] Singh. J.P., W.-D. Weber, and A. Gupta, SPLASH: Stanford Parallel Applications for Shared Memory. Technical Report CSL-TR-91-469. Stanford University. 1991.

[15] Xilinx. The Programmable Gate Array Data Book. 1991. 